Journal of Applied Fluid Mechanics, Vol. 4, No. 2, Special Issue, pp. 43-49, 2011. Available online at www.jafmonline.net, ISSN 1735-3572, EISSN 1735-3645. DOI: $10.36884 / \mathrm{jafm} .4 .03 .11933$

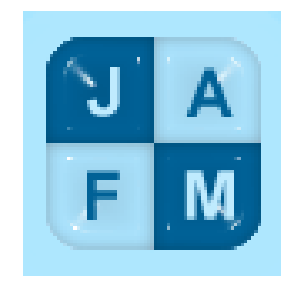

\title{
Modeling and Simulation of Interfacial Turbulent Flows
}

\author{
E. Shirani ${ }^{1 \dagger}$, F. Ghadiri ${ }^{1}$ and A. Ahmadi ${ }^{2}$ \\ ${ }^{I}$ Department of Mechanical Engineering, Isfahan University of Technology, \\ Isfahan, 84156-83111, Iran \\ ${ }^{2}$ Department of Mechanical Engineering, Majlesi Islamic Azad University, \\ Isfahan, Iran \\ $\dagger$ Corresponding Author Email: eshirani@ictp.it
}

(Received April 25, 2010; accepted March 13, 2011)

\begin{abstract}
Majority of the fluid flows in nature and industries are turbulent flows. Due to their complexity, modeling and simulation of turbulent flows are still among the top research topics in the field of fluid mechanics. The objective of this work is to consider the turbulence effects at the interface. The presence of interface affects the turbulence structures and they become anisotropic near the interface. In this work, the main objective is to consider the fluctuations of the interface topology and their effects on the volume fraction and the surface tension force at the interface. These effects are important under some circumstances especially when the shape of the interface changes rapidly and abruptly. The surface tension forces and the volume fraction-velocity fluctuation correlation have also important impact on the interface topology and its complicated features such as coalescence and breakup. Different new models are presented and the impacts of those parameters on the flow at the interface are presented in this work. In developing the models for mean velocity-volume fraction fluctuations the inhomogeneity of the flow at the interface is taken into account. Both Reynolds Averaged Navier-Stokes Equations and the Large Eddy Simulation Techniques were used to simulate turbulent interfacial flows and implement the novel models introduced in this work. The Kelvin-Helmholtz instability, two-dimensional and three dimensional jets, and water/oil phase separation were simulated numerically and the results were compared with corresponding valid data and the accuracy of the models was examined.
\end{abstract}

Keywords: Interfacial flows, Turbulrnce modelling, LES, VOF method.

\section{NOMENCLATURE}

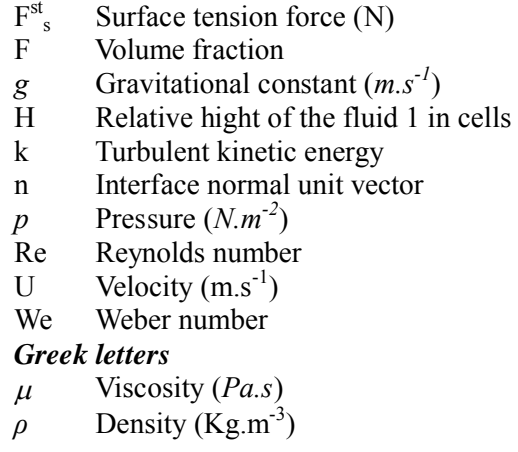

\section{INTRODUCTION}

Large number of flows in nature and industries involve free surfaces or material interfaces. Their applications range from environmental sciences, geophysics, and fundamental physics to numerous engineering problems. The shape of the interface as a sharp

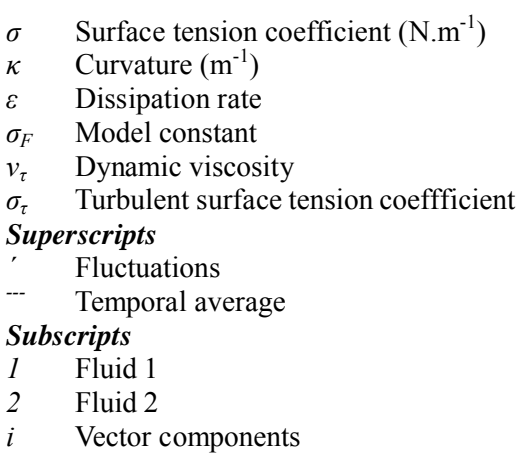

discontinuity plays an important role in dynamics of the problem. The accurate prediction of position, curvature and topology of the interface is essential in simulating the problem. Numerous methods have been developed to capture the interface motion. Almost all of methods are designed for laminar flows and no consideration is given to the turbulence effects. Therefore, using these 
methods without modifications to simulate turbulent free-surface flows cannot be a realistic and accurate treatment of the problem. The effect of turbulence on the interface can be quite significant. For example, the surface normal component of the turbulent kinetic energy may be redistributed into surface parallel component. This behavior, the anisotropy of turbulence near the interface, is due to the surface tension and the gravity forces depending on the flow Weber and Froude numbers, respectively. Study of the turbulent interfacial flows can be devided into the following two categories: first, experimental investigations of the effects of turbulence in flows with interface and second turbulence modeling and numerical simulation of such flows Turbulence modeling of flows with interfaces is in its early stages. Some of the main works in this area are described below.

Direct Numerical Simulation, DNS, technique, although is expensive and limited to simple and rather smooth interface topology, is used to study the turbulence of the interface. Among these studies, the analysis of energy balance and length scales of turbulence near the interface was studied by (Handler et al. 1993). (Komori et al. 1993) studied the flow at the interface with zero shear rates. Another investigation in this subject is the interaction of the waves and turbulence (Borue et al. 1995). The structures of turbulence near the interface of the gas-liquid (Lambardi et al. 1996) and turbulence at the interface of air -water shear flow at the $\mathrm{Re}=170$ (Falgosi et al. 2003) was also analyzed. Although DNS is a very strongh technique in the investigation of turbulence detailes, its applicability is limited to low Reynolds numbers, Re, as well as simple geometry and flow configuration. So the researches try to lower this cost by modeling the small eddies and resolving the large scales in LES method. Some of the recent investigations are as follows:

Channel flows with fully developed condition (Fureby et al. 1997) and with moderate Froude and Reynolds numbers (Shi et al. 2000) was studied using LES technique. Mass transfer at the air - water interface at high Schmit numbers was resolved by this method (Mitsobishi et al. 2003). Air entraiment under plunging breaking waves was numerically studied by LES simulation (Lubin et al. 2006). Interaction of turbulence and interface in bubbling process is another problem with interface that is investigated by LES method (Liovic and Lakehal 2007). While the computational costs of LES method in comparison to DNS method is less, this method is aplicabale for simulation of flows with limited interface deformation and Reynolds number. In addition none of the previous studies tried to derive the model for the turbulence term in averaging process in VOF equation and surface tension in momentum equations. RANS method was developed and used for the flows with interface by several researchers. In the next part some of the main works in this area are described.

A turbulent jet near a free surface by deriving a set of Reynolds averaged equations for free surface flows were studied by (Hong and Walker 2000). They showed that for low Froude numbers, the Reynolds stress anisotropy was mainly responsible for the outward acceleration of the surface current. For high Froude numbers, the Reynolds stress anisotropy was smaller and the free surface fluctuations made a significant contribution to the surface currents. Another set of transport equations for turbulent multiphase flows derived based on various forms of averaging (Banerjee 1990). The main investigation was that the turbulence structure near the gas-liquid interfaces depends primarily on the shear rate. In another study three algebraic stress models for predicting turbulent stresses near the free surface was presenred (Walker and Chen 1994). $k-\varepsilon$ model along with non-linear Reynolds stress model to simulate breaking waves in the surf zone was studied in another investigation (Lin and Liu 1998). None of the previous works on the turbulence modeling of interfacial flows include the effects of pressure fluctuations on the interface itself. Turbulent pressure fluctuations near the interface may generate very large local interface curvatures, resulting in large local surface forces at the interface. It may affect the breakup process. In the present study, two techniques of simulating interfacial flows, RANS and LES, were used for simulation of interfacial flows. A number of researchers (Klin and Janicka 2003; Herrmann 2003) have pointed out that as a result of averaging process (or filtering in the case of LES), some new terms appear in the resulting governing equations. The subgrid-scale surface tension term is mentioned, however, no model has been presented for it in his work. There are limited studies relevant to modeling turbulent interfacial flows using Reynolds- Averaged Navier-Stokes (RANS) and/or Large Eddy Simulation (LES) in the Eulerian formulation. The effect of pressure fluctuations and its correlation with the interface fluctuations was considered by (Shirani et al. 2006). In another study, LES methodology to multiphase flows was applied and has proposed relations for Large Scale Simulation (LSS) of these flows (Alajbegovic 2001). He has derived a general closure for the subgrid surface tension force. However, he has not provided the details of this closure term, since the relationship between the closure coefficient, the flow turbulence and the free-surface motion characteristics was not provided. In this paper, the sheared interface between two immiscible fluids at high Reynolds numbers and the spreading of plane water jet in air are simulated. This study is the continuation of our research group researches to simulate more complex turbulent interfacial flows. Our main objective is to introduce ideas and models of turbulence necessary to simulate interfacial flows. The correlation of the volume fraction fluctuations with velocity is modeled and the model is used in the volume fraction equation. The new models are applied in RANS formalism and LES. We have used our proposed models to simulate the primary breakup of immiscible fluid interfaces. Some model constants are obtained by comparing the simulation results of a plane turbulent water jet injected into still air with the experimental results (Sallam et al. 1999). The governing equations for two-dimensional unsteady, incompressible flows are used in the form of the Reynolds averaged equations. To close the time averaged governing equations, three different turbulence models along with the new models for the fluctuation of the pressure interface location and the volume fraction-velocity correlations are used. It is assumed that the fluids are immiscible without phase 
change and the Volume-Of-Fluid (VOF) method is used for capturing the interface motion.

\section{GOVERNING EQUATIONS AND NUMERICAL METHODS}

2D and 3D incompressible time dependent Navier-Stokes equations for a two-fluid problem including the liquid interfaces are used. It is assumed that the velocity field is continuous across the interface, but there is a pressure jump at the interface due to the surface tension. Following the VOF method, the advective equation for volume fraction, $F$, is used to calculate the volume fraction and the shape of the interface. The following different techniques are used here for modeling the turbulent interfacial flows.

\subsection{RANS Equations}

Assuming that the time scale of the turbulent flows is small compared to the time scale of the mean flow structures at the interface, the time average of the governing equations are as follow.

$$
\begin{aligned}
& \partial \bar{u}_{i} / \partial x_{i}=0 \\
& \frac{\partial \bar{F}}{\partial t}+\frac{\partial \bar{u}_{i} \bar{F}}{\partial x_{i}}+\frac{\partial \overline{u_{i}^{\prime} F^{\prime}}}{\partial x_{i}}=0 \\
& \frac{\partial \bar{u}_{i}}{\partial t}+\frac{\partial \bar{u}_{i} \bar{u}_{j}}{\partial x_{j}}+\frac{\partial \overline{u_{i}^{\prime} u_{j}^{\prime}}}{\partial x_{j}}= \\
& -\frac{1}{\bar{\rho}} \frac{\partial \bar{P}}{\partial x_{i}}+\frac{\bar{\mu}}{\bar{\rho}} \frac{\partial^{2} \bar{u}_{i}}{\partial x_{j} \partial x_{i}}+\frac{1}{\bar{\rho}} \overline{F_{i}^{s t}}
\end{aligned}
$$

In general, $F_{i}{ }^{s t}$ can be given as $\sigma \kappa \delta_{s} n_{i}$. The time averages of density and viscosity are:

$$
\begin{aligned}
& \bar{\rho}=\rho_{2}+\bar{F}\left(\rho_{2}-\rho_{1}\right) \\
& \bar{\mu}=\mu_{2}+\bar{F}\left(\mu_{2}-\mu_{1}\right)
\end{aligned}
$$

respectively. Due to the fluctuations of the pressure and velocity in the interface region and the motions of eddies in this region, the interface location may fluctuate. Thus, the curvature of the interface is a turbulent quantity and it fluctuates with time and space. In fact, the fluctuation of the interface location is related to the pressure fluctuations in this region. This suggests that the pressure and the interface location fluctuations may be well correlated and would affect the flow characteristics at the interface. The pressure in the interfacial region with the presence of surface tension needs careful consideration. In fact, due to the sharp discontinuity of the pressure at the interface, the pressure force in the interfacial cells depends on the location of the interface at the cell faces. So, in these equations the unkown termes are:

$\overline{u_{i}^{\prime} u_{j}^{\prime}}, \bar{P}, \overline{F^{\prime} u_{i}^{\prime}}$

The first unkown term is the Reynolds stress. To model the first term, $k-\varepsilon$ based model which consists of a realizable Reynolds stress algebraic equation model is used (Shih et al. 1995). The model has significantly improved the predictive capability of $k$ - $\varepsilon$ based models, especially for flows involving strong shear layers. The equations for these models are presented as below.

$\frac{\partial k}{\partial t}+\bar{u}_{j} \frac{\partial k}{\partial x_{j}}=\frac{\partial}{\partial x_{j}}\left(\left(v+\frac{v_{\tau}}{\sigma_{k}}\right) \frac{\partial k}{\partial x_{j}}\right)-\overline{u_{i}^{\prime} u_{j}^{\prime}} \frac{\partial \bar{u}_{i}}{\partial x_{j}}-\varepsilon$

$\frac{\partial \varepsilon}{\partial t}+\bar{u}_{j} \frac{\partial \varepsilon}{\partial x_{j}}=\frac{\partial}{\partial x_{j}}\left(\left(v+\frac{v_{\tau}}{\sigma_{k}}\right) \frac{\partial \varepsilon}{\partial x_{j}}\right)$

$-\mathrm{C}_{\varepsilon 1} \frac{\varepsilon}{k} \overline{u_{i}^{\prime} u_{j}^{\prime}} \frac{\partial \bar{u}_{i}}{\partial x_{j}}-\mathrm{C}_{\varepsilon 2} \frac{\varepsilon^{2}}{k}$

where $\sigma_{k}=1, \sigma_{\varepsilon}=1.3, C_{\varepsilon 1}=1.44$, and $C_{\varepsilon 2}=1.92$. The Reynolds stresses can be written as:

$\overline{u_{i}^{\prime} u_{j}^{\prime}}=\frac{2}{3} k \delta_{i j}-2 C_{\mu} \frac{k^{2}}{\varepsilon} S_{i j}+2 C_{2} \frac{k^{3}}{\varepsilon^{2}}\left(S_{i k} \Omega_{k j}+S_{k j} \Omega_{i k}\right)$

and the kinematic eddy viscosity, $v_{\tau}$, is related to $k$ and $\varepsilon$, according to

$v_{\tau}=C_{\mu} k^{2} / \varepsilon$

where, $C_{\mu}=0.09$ and $C_{2}=0$ for standard $k-\varepsilon$ model. For the algebraic model, these coefficients are:

$$
\begin{aligned}
& S_{i j}=\frac{1}{2}\left(\frac{\partial u_{i}}{\partial x_{j}}+\frac{\partial u_{j}}{\partial x_{i}}\right)-\frac{1}{3} \frac{\partial u_{k}}{\partial x_{k}} \delta_{i j} \\
& \Omega_{i j}=\frac{1}{2}\left(\frac{\partial u_{i}}{\partial x_{j}}-\frac{\partial u_{j}}{\partial x_{i}}\right) \quad C_{\mu}=\left(6.5+A \frac{U k}{\varepsilon}\right)^{-1} \\
& C_{2}=\sqrt{1-9 S_{i j} S_{i j} C_{\mu}^{2}\left(\frac{k}{\varepsilon}\right)^{2}} /\left(1+6 \frac{\sqrt{S_{i j} S_{i j}} k}{\varepsilon} \frac{\sqrt{\Omega_{i j} \Omega_{i j}} k}{\varepsilon}\right) \\
& A=\sqrt{6} \operatorname{Cos}(\operatorname{Arc} \cos (\sqrt{6} W) / 3) \\
& W=S_{i j} S_{j k} S_{k i} /\left(S_{i j} S_{i j}\right)^{3 / 2} \quad U=\sqrt{S_{i j} S_{i j}+\Omega_{i j} \Omega_{i j}}
\end{aligned}
$$

The second term is the averaged pressure. According to Fig.1, a relation between pressure at the cell center and the interface location in the same cell for one-dimensional case is

$$
p_{L}=p_{2 L}+H_{L}\left(p_{1 L}-p_{2 L}\right)
$$

The pressure difference in the parenthesis represents the pressure jump due to the surface tension. Similar relations can be obtained for the pressure force on the right, top and bottom sides. In general,

$$
p=p_{2}+H\left(p_{1}-p_{2}\right)
$$

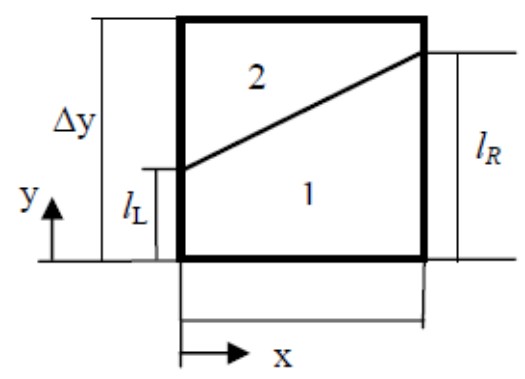

Fig. 1. a cell with an interface 
The time average of the pressure, Eq. (8), is:

$$
\bar{p}=\bar{p}_{2}+\bar{H}\left(\bar{p}_{1}-\bar{p}_{2}\right)+\overline{H^{\prime}\left(p_{1}^{\prime}-p_{2}^{\prime}\right)}
$$

The second term on the right hand side of Eq. (9) corresponds to the mean values and it is related to the molecular surface tension:

$$
\bar{H}\left(\bar{p}_{1}-\bar{p}_{2}\right)=\sigma \bar{\kappa} \bar{H}
$$

The last term in Eq. (9) introduces a mean value of fluctuating quantities which needs to be modeled. This term is responsible for the effect of pressure fluctuations at the interface, which may affect the breakup process and the generation of disturbances and waves at the interface. This term by two ways of scale analysis and dimentional analysis was modeled (Shirani 2006). The statement below show the magnitude of unkonown term by scale analysis:

$$
\overline{\left(p_{1}^{\prime}-p_{2}^{\prime}\right) H^{\prime}}=\sigma \overline{\kappa^{\prime} H^{\prime}}=C_{p}^{\prime} \sigma \bar{\kappa}\left(\mu_{\tau} / \mu\right)^{1 / 2}
$$

Another approximation for this term that was derived by dimentional analysis and presented by equation below:

$\overline{\left(p_{1}^{\prime}-p_{2}^{\prime}\right) H^{\prime}}=\sigma_{\tau} \bar{\kappa} \bar{H}=C_{p}^{\prime \prime} \sigma \bar{\kappa}\left(\mu_{\tau} / \mu\right)^{1 / 2} \bar{H}$

These models are new and considered for the first time. $C_{p}^{\prime}$ and $C_{p}^{\prime \prime}$ are model constants. Comparing (11) and

(12), it is observed that they are basically similar except for the $H$ term. It is shown that the surface tension term increases in turbulent flows. This magnification is either represented by the fluctuations in curvature, with molecular surface tension coefficient or by turbulent surface tension coefficient with an average curvature. In both cases, the expressions are nearly identical.

The last indefinite term in RANS formulation for interfacail flows is $\overline{F^{\prime} u_{i}^{\prime}}$. The volume fraction-velocity correlation can be modeled similar to that of Bosinesque approximation. However, the simple gradient transport is only appropriate for homogeneous flows, where the size of the energy-containing eddies is smaller than the distance over which the gradient varies appreciably. Most of the turbulent interfacial flows are intermittent flows like jet flow that one fluid in turbulent regim and onother one in laminar regime. In this case the velocity-volume fraction corolation term are modelled as a turbulent intermittent flow (Byggstoyl and Kollmann 1981) and offered by equation below:

$$
-\overline{F^{\prime} u_{i}^{\prime}}=\sigma_{F}(1-\bar{F}) \mu_{\tau} / \rho \partial \bar{F} / \partial x_{i}
$$

\subsection{LES Equations}

In another modeling process the filtering method was used for LES method. In this filtering method the small eddies are modeled and large eddies are resolved directly. The filtered equations for large eddies are as follows:

$$
\partial \bar{u}_{i} / \partial x_{i}=0
$$

$$
\begin{aligned}
& \frac{\partial \bar{F}}{\partial t}+\frac{\partial \bar{u}_{i} \bar{F}}{\partial x_{i}}=\frac{\partial\left(\bar{u}_{\mathrm{i}} \bar{F}-\overline{u_{i} F}\right)}{\partial x_{i}} \\
& \frac{\partial \bar{u}_{i}}{\partial t}+\frac{\partial \bar{u}_{i} \bar{u}_{j}}{\partial x_{j}}=-\frac{1}{\bar{\rho}} \frac{\partial \bar{P}}{\partial x_{i}}+\frac{\bar{\mu}}{\bar{\rho}} \frac{\partial^{2} \bar{u}_{i}}{\partial x_{j} \partial x_{i}}+\frac{1}{\bar{\rho}} \sigma \bar{\kappa} \bar{n}_{i} \\
& +\frac{1}{\bar{\rho}}\left(\sigma \overline{\kappa n_{i}}-\sigma \bar{\kappa} \bar{n}_{i}\right)-\frac{\partial\left(\overline{u_{i} u_{j}}-\bar{u}_{i} \bar{u}_{j}\right)}{\partial x_{j}}+g_{i}
\end{aligned}
$$

The unkown term in the LES equations are:

$$
\overline{u_{i} u_{j}}-\bar{u}_{i} \bar{u}_{j}, \overline{\kappa n_{i}}-\bar{\kappa} \bar{n}_{i}, \bar{u}_{i} \bar{F}-\overline{u_{i} F}
$$

By using the approximations that used in the first part for RANS equations and developed for the LES equations these unkown terms can be defined by the estimation below:

The first unkown term is Reynolds stress subgrid scales and estimated by the smagorinsky model:

$$
\overline{u_{i} u_{j}}-\bar{u}_{i} \bar{u}_{j}=-\frac{1}{2} \rho v_{t} S_{i j}
$$

Where

$$
v_{\tau}=l^{2}|\bar{S}| \quad l=C_{s} \Delta \quad|\bar{S}|=\sqrt{2 S_{i j} S_{i j}}
$$

In this model $l$ is the length a scale of small eddies and $C_{s}$ is the smagorinsky constant.

The second unkown term is $\overline{\kappa n_{i}}-\bar{\kappa} \bar{n}_{i}$.this term can be modeled like dimentional analysis for the pressure term in RANS equations.

$\overline{\kappa n_{i}}-\bar{\kappa} \bar{n}_{i}=C_{s t} \sqrt{|\bar{S}|} \bar{\kappa} \bar{n}_{i} \Delta / \sqrt{v}$

$C_{s t}$ is a new model constant like smagorinsky model constant.

The last unkown term is $\bar{u}_{i} \bar{F}-\overline{u_{i} F}$. This term modeled by the approximation like the model for volume fraction-velocity corolation in RANS equations and developed for LES model as follow:

$$
\bar{u}_{i} \bar{F}-\overline{u_{i} F}=C_{F} \bar{F}(1-\bar{F}) \Delta^{2}|\bar{S}| \partial F / \partial x_{i}
$$

$C_{F}$ is new model constant like smagorinsky model constant. Here in the present study, a VOF method based on Piecewise Linear Interface Calculation (PLIC) along with a projection method to solve the 2-D unsteady incompressible Navier-Stokes equations on a staggered grid and the continuous surface stress (CSS) method for modeling the interfacial tension is used in this work. The computational grid is fixed, rectangular and uniform. The code SURFER for RANS simulations and a research code for LES is modified and used in this work. In the next section the results of these new models implemention in computer code will be presented.

\section{RESULTS AND DISCUSSIONS}

In the previous section two sets of equations and their unkown termes and correcponding turbulence models were introduced. Here the results devide into two parts; first implemention of new modles for RANS method and second implemention of new modles for LES scheme. 


\subsection{RANS Method}

To examine these sets of equations, they are implemented in 2D incompressible time dependent flow and two cases were examined by this new code, first, Kelvin-Helmholtz instability problem and second, to find the appropriate model constant the 2D plane jet of water in still air that its experimental results are valid.

We first consider a sheared immiscible interface between two fluids of equal densities and viscosities. The initial configuration is shown in Fig.2. Domain size is $2 \lambda$ in both horizontal and vertical directions.

Boundary conditions are periodic in the horizontal direction and free-slip at the top and the bottom. The fluid below the interface is moving to the right with velocity $\Delta U / 2$ and the fluid on the top of the interface moves to the left with velocity $\Delta U / 2$. The initial perturbation amplitude is equal to $10 \%$ of the wavelength of the initial perturbation. The Reynolds and Weber numbers are defined based on the wavelength as $R e=\rho \Delta U \lambda / \mu$ and $W e=\rho(\Delta U)^{2} \lambda /(2 \pi \sigma)$. The values of $R e$ and $W e$ numbers are $5 \times 10^{5}$ and 150 , respectively.

A $128 \times 128$ computational grid is used. Time is nondimentionalize by velocity difference of two fluid and initial wave length. Fig. 3 shows the interface topology for time 6.5 that the ligament of fluid 1 starts to break for different model constant. Results show that this term shows its effect in large magnitude (more than 20).

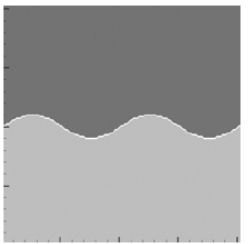

Fig. 2. Initial flow field

For the second case the Kelvin-Helmholts instability was studied for two fluids that the upper one at rest and the lower one moves to right direction. Re and We numbers are like the case. Results in Fig. 4 show that the effect of the new term for of velocity-volume fraction flactuations correlation for large model constat becomes important. The breakup of the ligament takes place sooner and shape of the interface for this case is almost similar to previous case.

For the third case the Kelvin-Helmholts instability was studied for two fluids that the upper one at rest and the lower one moves to right direction. The conditions are the same as two cases but the fluid 1 has the properties of water and the fluid 2 has the properties of air. Results show the effect of density ratio for two fluids at time 6 , Fig. 5.

To find the best value for $\sigma_{F}$ (model constant) the code was set for simulation of $2 \mathrm{D}$ plane jet that its experimental data is valid. Result in Fig.6 show that $\sigma_{F}=20$ has a better compatability with experiments.

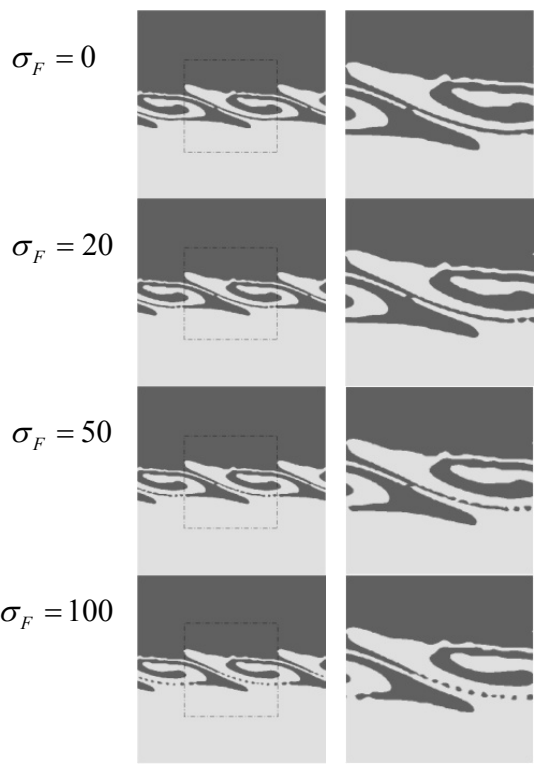

Fig. 3. Interface topology at nondimentional time $=6.5$

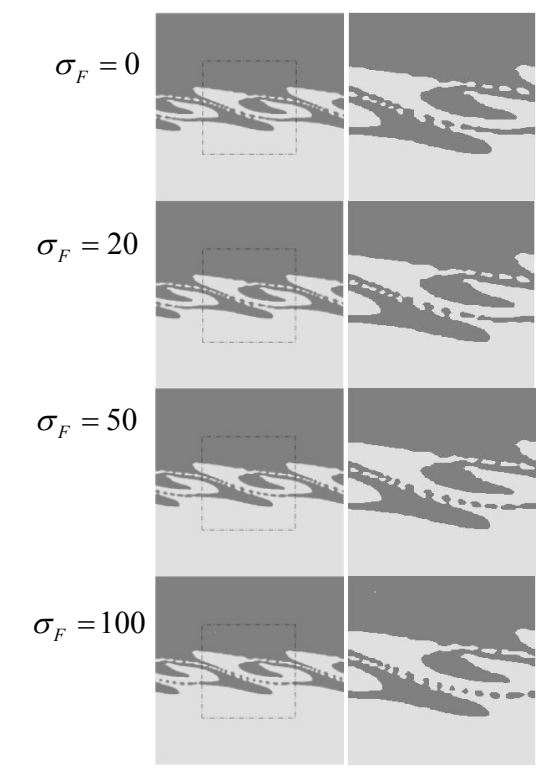

Fig. 4. Interface topology at nondimentional time $=5$

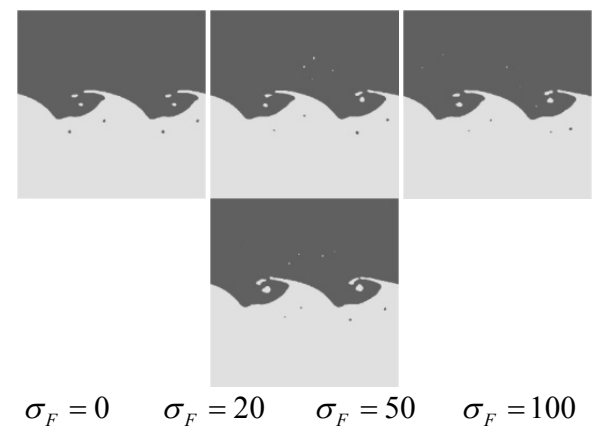

Fig. 5. Interface topology at nondimentional time $=6$ 


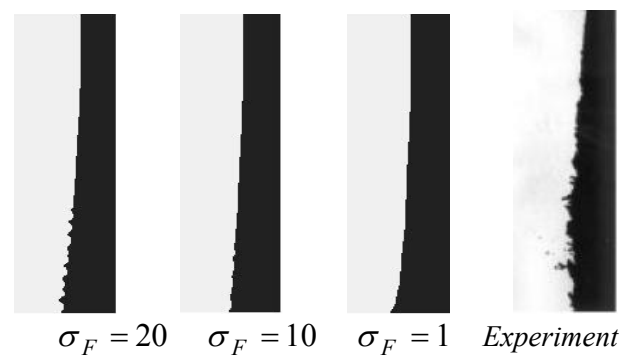

Fig. 6. Numerical and experimental results for plane jet spreading

\subsection{LES Method}

To examine the sets of equations, they are implemented in 3D incompressible time dependent flow and the case of oil columne collapsing in water that its DNS results are exist, was studied by developed code. The dimentions of the domain in three coordinates are $1 \mathrm{~m}$ and number of cells in each direction is 64. By this computaioal domain the size of each cell is $0.015 \mathrm{~m}$ that is 2.5 times greater that the Taylor length scales. Interface profile for the both LES results and DNS results are shown in Fig 7. In this case the $C_{F}$ is equal to 0.45 and $C_{s t}$ eaual to 0.15 .
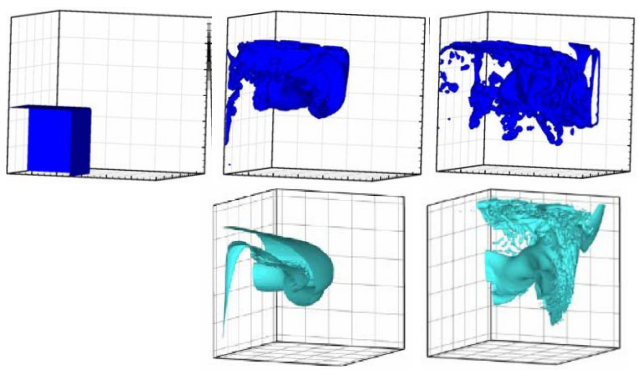

$\mathrm{t}=0 \mathrm{~s}$

$\mathrm{t}=2 \mathrm{~s}$

$\mathrm{t}=4 \mathrm{~s}$

Fig. 7. Comparison of LES (first row) and DNS

(second row) results for oil column falling in water

The result of LES for turbulent kinetic energy of the water in compare with DNS is presented in Fig. 8. The figure shows good agreement between DNS and LES results.

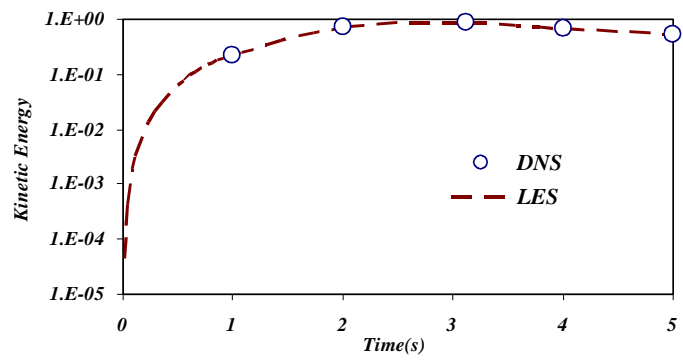

Fig. 8. Comparison of LES turbulent kinetic energy of water with DNS results

The results of LES simulation in present work for normalized advection term in averaged transport equation is shown in Fig. 9. The figure shows that this term with the magnitude of $58 \%$ at time equal to $5 \mathrm{sec}$ has significant effect in prediction of surface topology and is not negligible.

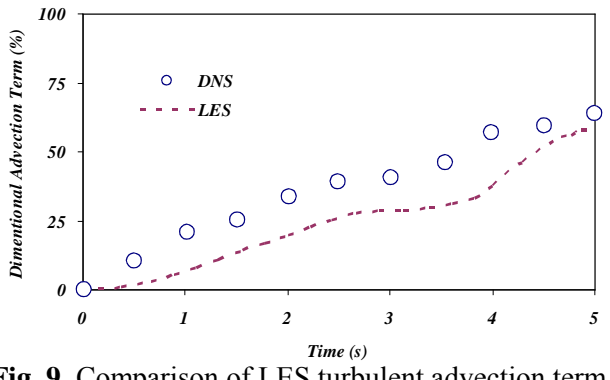

Fig. 9. Comparison of LES turbulent advection term with DNS results

Figure 10 shows the comparison of turbulent surface tension presented in this work with DNS results in both direction of $\mathrm{X}$ and $\mathrm{Y}$. The magnitudes of these terms were normalized with maximum value in each direction. By evaluation of these term it is derived that although the flow in $\mathrm{Y}$ direction is anisotropic (because of gravity and boyancy), the magnitudes of this term in both directions are almost the same and the anisotropy of the flow doesn't have any effect on this term. LES simulation couldn't predict this trend. Note that the DNS results shown in Figs. 7 to 10 are taken from Vincent et al., 2006 and Vincent et al., 2008 and the experimental results shown in Fig. 6 is taken from Sallam et al., 1999.

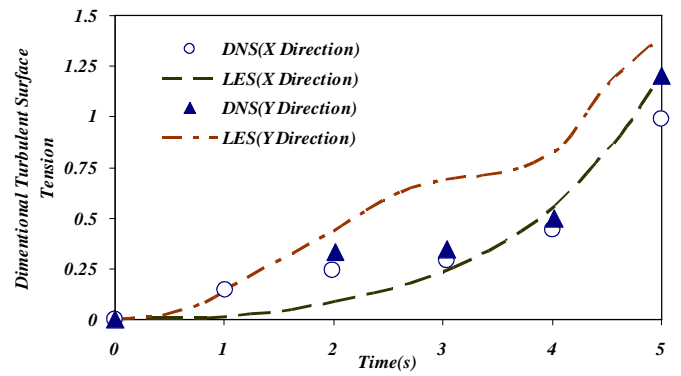

Fig. 10. Comparison of LES turbulent surface tension term with DNS results

\section{Conclusions}

In the present work the turbulent interfacial flows was studied. Equations for both LES and RANS simulations are presented and new terms that produced by averaging and filtering process in the equations are modelled. By comparing the results of these simulation with experimental and DNS results the appropriate magnitude of model constants were derived. Results show that the new terms introduced and modelled in this work have a significant effect in simulation results and can not be neglected.

\section{REFERENCES}

Alajbegovic, A. (2001). Large Eddy Simulation Formalism Applied to Multiphase Flows. ASME FEDSM'01, USA.

Banerjee, S. (1990). Modeling Considerations for Turbulent Multiphase Flows. Engineering 
Turbulence Modeling and Experiments, Rodi and Ganic (eds.), 831-866.

Borue. V., S.A. Orszag and I. Staroselsky (1995). Interaction of surface waves with turbulence direct numerical simulations of turbulent open-channel flow. J. Fluid Mech. 286, 1-23.

Byggstoyl, S., and W. Kollmann (1981). Closure Model for Intermittent Turbulent Flows. J. Heat Mass Transfer 24(11), 1811-1822.

Fulgosi, D., S. Lakehal, V. Banerjee, and De Angelis (2003). Direct numerical simulation of turbulence in sheared air-water flow with a deformable interface. J. Fluid Mech. 482, 319-345.

Fureby, C., A.D. Gosman, G. Tabor, G. Weller, N. Sandham, M. Wolfshtein (1997). Large eddy simulation of turbulent channel flows. Turbulent Shear Flows 11.

Handler, R.A., T.F. Swean, R.I. Leighton and J.D. Swearingen (1993). Length scales and energy balance for turbulence near a free surface. AIAA J. 31, 1998-2007.

Herrmann, M. (2003). Modeling Primary Breakup: a Three-Dimensional Eulerial Level Set/Vortex Sheet Method for Two-Phase Interface Dynamics. Annual Research Briefs, Center for Turbulence research, Stanford University, 185-196.

Hong, W., D.T. Walker (2000). Reynolds-Averaged Equations for Free-Surface Flows with Application to High-Froude-Number Jet Spreading. J. Fluid Mech. 417,183-209.

Klein, M., and J. Janicka (2003). Large-Eddy Simulation of the Primary Breakup of a Spatially Developing Liquid Film. ICLASS, Sorrento, Italy

Komori, S., R. Nagaosa, Y. Murakami, S. Chiba, K. Ishii, and K. Kuwahara (1993). Direct numerical simulation of three-dimensional open-channel flow with zero-shear gas-liquid interface. Phys. Fluids, 115-125.

Lin, P., and P.A. Liu (1998). Numerical Study of Breaking Waves in the Surf Zone. J. Fluid Mech. 359, 239-264.

Liovic, P., and D. Lakehal (2007). Interface-turbulence interactions in large-scale bubbling processes. Int. J. Heat and Fluid Flow 28, 127-144.

Lombardi, P., De Angelis, and S. Banerjee (1996). Direct numerical simulation of near-interface turbulence in coupled gas-liquid flow. J. Phys. Fluids 8(6), 1643- 1665.

Lubin, P., S. Vincent, S. Abadie, J.P. Caltagirone (2006). Three dimensional Large Eddy Simulation of air entrainment under plunging breaking waves, Coastal Engineering 53, 631-655.
Mitsubishi, A, Y. Hasegawa, and N. Kasagi (2003). Large eddy simulation of mass transfer across and air-water interface at high Schmidt numbers. The 6th ASME-JSME Thermal Engineering Joint Conference.

Sallam, K.A., Z. Dai, and G.M. Faeth (1999). Drop Formation at the surface of plane turbulent liquid jets in still gases. Int. J. Multiphase Flow 25, 1161-1180.

Shi, J., T.G. Thomas, and J.J.R. Williams (2000). Free-surface effects in open channel flow at moderate Froude and Reynolds number. $J$. Hydraulic Research, 38-6.

Shih, T.H., J. Zhu, and J.L. Lumley (1995). A New Reynolds Stress Algebraic Equation Model. Comput. Methods Appl. Mech. Engrg. 125, 287-302.

Shirani, E., A. Jafari, and N. Ashgriz (2006). Turbulence Models for Flows with Free Surfaces and Interfaces. AIAA. J. 44(7).

Vincent, S., Larocque, J., Lacanette, D., Toutant, A., Lubin, P. and Sagaut, P. (2008). Numerical Simulation of Phase Separation and a Priori Two-Phase LES Filltering, Computers \& Fluids Journal, 37, 898-906.

Vincent, S., Larocque, J.D., Lacanette, A., Toutant, P., Lubin, O., Lebaigue, E., Labourasse, J.-P. Caltagirone and P. Sagaut (2006) Governing Equations and a Priori Tests for the LES of Two-Phase Flows. Conference on Turbulence and Interactions TI2006.

Walker, D.T., and C.Y. Chen (1994). Evaluation of Algebraic Stress Modeling in Free-Surface Jet Flows, Free-surface turbulence. ASME, 181. 